\author{
K.K. Sadykova ${ }^{1,2}$, N.T. Tleukhanova ${ }^{1}$ \\ ${ }^{1}$ L.N. Gumilyov Eurasian National University, Nur-Sultan, Kazakhstan; \\ ${ }^{2}$ S. Seifullin Kazakh Agro Technical University, Nur-Sultan, Kazakhstan \\ (E-mail: sadkelbet@gmail.com)
}

\title{
Estimates of the norm of the convolution operator in anisotropic Besov spaces with the dominated mixed derivative
}

In this paper, we investigate the boundedness of the norm of the convolution operator in Sobolev spaces with the dominated mixed derivative and anisotropic Nikolsky-Besov spaces. For Sobolev spaces with the dominated mixed derivatives, an analogue of Young's inequality is obtained, namely, relations of the form

$$
W_{\mathbf{p}}^{\boldsymbol{\gamma}} * W_{\mathbf{r}}^{\boldsymbol{\beta}} \hookrightarrow W_{\mathbf{q}}^{\alpha}
$$

are proved when the corresponding conditions on the parameters are satisfied. The main goal of the paper is to solve the following problems. Let $f$ and $g$ be functions from some classes of the Nikolsky-Besov space scale. We would like to find the Nikolsky-Besov space such that the convolution $f * g$ belongs to this space. Using relation (1) and the Nursultanov interpolation theorem for anisotropic spaces, an analogue of the O'Neil theorem was obtained for the Nikolsky-Besov space scale $B_{\mathbf{p q}}^{\boldsymbol{\alpha}}$, where $\boldsymbol{\alpha}, \mathbf{p}, \mathbf{q}$ are vector parameters. Relations of the form $B_{\mathbf{p s}_{1}}^{\gamma} * B_{\mathbf{r s}_{2}}^{\boldsymbol{\beta}} \hookrightarrow B_{\mathbf{q s}^{\alpha}}^{\boldsymbol{\alpha}}$ are obtained, with the corresponding ratios of vector parameters. The theorems obtained in this paper complement the results of Batyrov and Burenkov, where similar problems were considered in isotropic Nikolsky-Besov spaces, that is, in spaces where the parameters are scalars.

Keywords: convolution operator, anisotropic Sobolev and Besov spaces, interpolation.

\section{Introduction}

Let $I$ be either a $n$-dimensional torus $\mathbb{T}^{n}=[0,1)^{n}$, or the Euclidean space $\mathbb{R}^{n}$. Let $f(x)$ and $g(x)$ be measurable functions on $I$ with respect to the $n$-dimensional Lebesgue measure such that for almost all $x \in I$ there exists an integral

$$
\int_{I} f(x-y) g(y) d y .
$$

In this case, it is said that the convolution of these functions is defined

$$
(f * g)(x)=\int_{I} f(x-y) g(y) d y .
$$

The classical Young's inequality $[1 ; 199]$ has the following form. Let

$$
1 \leq p, r, q \leq \infty, \quad \frac{1}{q}+1=\frac{1}{p}+\frac{1}{r} .
$$

If $f \in L_{p}(I), g \in L_{r}(I)$, then there exists almost everywhere on $I$ the convolution $f * g$, belonging to the space $L_{q}(I)$ and the following inequality holds

$$
\|f * g\|_{L_{q}(I)} \leq\|f\|_{L_{p}(I)}\|g\|_{L_{r}(I)}
$$

We will write this statement as follows

$$
L_{p}(I) * L_{r}(I) \hookrightarrow L_{q}(I) .
$$


This inequality plays an important role in harmonic analysis and in the theory of partial differential equations $[1-3]$.

Note that if

$$
1<p, r, q<\infty, \quad \frac{1}{q}+1=\frac{1}{p}+\frac{1}{r},
$$

then for $g_{0}(x)=\frac{1}{|x|^{\frac{n}{r}}}$ the inequality holds

$$
\left\|f * g_{0}\right\|_{L_{q}(I)} \leq C\|f\|_{L_{p}(I)} .
$$

This inequality is called the Hardy-Littlewood-Sobolev inequality. It does not follow from Young's inequality, since $\left\|g_{0}\right\|_{L_{r}(I)}=\infty$. A generalization of inequality (1.3) obtained by O'Neil [4] (see also [5, 6]).

If (1.4) is true and $0<s_{1}, s_{2}, s \leq \infty, \frac{1}{s}=\frac{1}{s_{1}}+\frac{1}{s_{2}}$, then

$$
L_{p s_{1}} * L_{r s_{2}} \hookrightarrow L_{q s}
$$

and in particular

$$
L_{p} * L_{r \infty} \hookrightarrow L_{q}
$$

where $L_{p s}$ is Lorentz space.

Note that in relation (1.5), condition (1.4) is essential. The limiting cases of the O'Neil inequality with condition (1.2) were considered in [7].

The O'Neil inequality for anisotropic Lorentz spaces was studied in [8-10]. In the case of $n \geq 2$ these results are extend the inequality (1.6). In the one-dimensional case, the O'Neil inequality was extended in [11, 12].

There are generalizations of the Young and O'Neil inequalities for various functional spaces: weighted $L_{p}$ spaces, classical and Lorentz weighted spaces, Hardy spaces, Wiener spaces, Orlicz spaces; [5, 6, 8, 13-18], and references therein.

Convolution operators were studied in various spaces of smooth functions in [19-22].

V.I. Burenkov and B.E. Batyrov in [21] proved the following statement: let $-\infty<l_{1}, l_{2}, l_{3}<\infty$, $0<p_{1}, p_{2}, p_{3} \leq \infty, 0<\theta_{1}, \theta_{2}, \theta_{3} \leq \infty$. For any $f_{1} \in B_{p_{1} \theta_{1}}^{l_{1}}\left(\mathbb{R}^{n}\right), f_{2} \in B_{p_{2} \theta_{2}}^{l_{2}}\left(\mathbb{R}^{n}\right)$ such that $F f_{1}$ and $F f_{2}$ are regular generalized functions and their (pointwise) product $F f_{1} \cdot F f_{2} \in S\left(\mathbb{R}^{n}\right)$, there exists a number $c_{3}>0$ such that

$$
\left\|f_{1} * f_{2}\right\|_{B_{p_{3} \theta_{3}}^{l_{3}}\left(\mathbb{R}^{n}\right)} \leq c_{3}\left\|f_{1}\right\|_{B_{p_{1} \theta_{1}}^{l_{1}}\left(\mathbb{R}^{n}\right)}\left\|f_{2}\right\|_{B_{p_{2} \theta_{2}}^{l_{2}}\left(\mathbb{R}^{n}\right)}
$$

holds if and only if the following conditions hold:

1) $p_{3} \geq p_{1}, p_{3} \geq p_{2}$;

2) $\frac{1}{p_{1}}+\frac{1}{p_{2}}-\frac{1}{p_{3}}-1 \geq 0$;

and one of the conditions

or

3a) $l_{3}<l_{1}+l_{2}-n\left(\frac{1}{p_{1}}+\frac{1}{p_{2}}-\frac{1}{p_{3}}-1\right)$

3b) $l_{3}=l_{1}+l_{2}-n\left(\frac{1}{p_{1}}+\frac{1}{p_{2}}-\frac{1}{p_{3}}-1\right)$ и $\frac{1}{\theta_{3}} \leq \frac{1}{\theta_{1}}+\frac{1}{\theta_{2}}$,

where $F f$ is the Fourier transform of the function $f$ :

$$
(F f)(x)=(2 \pi)^{-\frac{n}{2}} \int_{\mathbb{R}^{n}} e^{-i x \xi} f(\xi) d \xi .
$$

For $p_{2}=p_{3}, \theta_{2}=\theta_{3}, 0<l_{2}<l_{3}<\infty$ inequality (1.7) and some of its generalizations follow from the results obtained in the works of K.K. Golovkin and V.A. Solonnikov [19, 20], and [23].

In this paper, we investigate the boundedness of the convolution operator in anisotropic Besov spaces with the dominated mixed derivative. 


\section{Anisotropic Besov spaces with dominated mixed derivative}

Let $\boldsymbol{\alpha} \in \mathbb{R}^{n}, \mathbf{1}<\mathbf{p}=\left(p_{1}, \ldots, p_{n}\right)<\infty, \mathbf{0}<\mathbf{q}=\left(q_{1}, \ldots, q_{n}\right) \leq \infty$. Following [24-26], we define the space $B_{\mathbf{p q}}^{\boldsymbol{\alpha}}\left(\mathbb{T}^{n}\right)$ as the set of series $f=\sum_{m \in \mathbb{Z}^{n}} a_{m} e^{2 \pi i(m, x)}$ (generally speaking, divergent) for which

$$
\|f\|_{B_{\mathbf{p q}}^{\alpha}\left(\mathbb{T}_{n}\right)}=\left(\sum_{k_{n}=0}^{\infty} \cdots\left(\sum_{k_{1}=0}^{\infty}\left(2^{\sum_{j=1}^{n} \alpha_{j} k_{j}}\left\|\Delta_{k}(f)\right\|_{L_{\mathbf{p}}\left(\mathbb{T}^{n}\right)}\right)^{q_{1}}\right)^{\frac{q_{2}}{q_{1}}} \cdots\right)^{\frac{1}{q_{n}}}<\infty
$$

is finite, where $\Delta_{k}(f)(x)=\sum_{\substack{2^{k_{j}-1} \leq\left|m_{j}\right|<2 \\ j=1, \ldots, n}} a_{m} e^{2 \pi i(m, x)}, k \in \mathbb{Z}_{+}^{n},(m, x)=\sum_{i=1}^{n} m_{i} x_{i}$.

For $q=\infty$ the values of $\left(\sum_{k \in \mathbb{Z}} b_{k}^{q}\right)^{\frac{1}{q}},\left(\int_{\mathbb{T}} f^{q}\right)^{\frac{1}{q}}$ are understood respectively as $\sup _{k \in \mathbb{Z}}\left|b_{k}\right|$, ess $\sup _{x \in \mathbb{T}}|f(x)|$.

We say that the series $f=\sum_{k \in \mathbb{Z}^{n}} a_{k} e^{2 \pi i(k, x)}$ is an element of the space $W_{\mathbf{p}}^{\boldsymbol{\alpha}}\left(\mathbb{T}^{n}\right)[24]$ if there is a function $f^{\alpha} \in L_{\mathbf{p}}\left(\mathbb{T}^{n}\right)$ Fourier series of which coincides with the series $\sum_{k \in \mathbb{Z}^{n}} \bar{k}^{\alpha} a_{k} e^{2 \pi i(k, x)}$, here $\bar{k}^{\alpha}=\prod_{j=1}^{n} \bar{k}_{j}^{\alpha}$, $\bar{k}_{j}=\max \left\{\left|k_{j}\right|, 1\right\}, j=1, \ldots, n$,

$$
\|f\|_{W_{\mathbf{p}}^{\alpha}\left(\mathbb{T}^{n}\right)} \stackrel{\operatorname{def}}{=}\left\|f^{\alpha}\right\|_{L_{\mathbf{p}}\left(\mathbb{T}^{n}\right)}
$$

We define the concept of convolution for the elements of these spaces.

Let $f=\sum_{k \in \mathbb{Z}^{n}} a_{k} e^{2 \pi i(k, x)}$ and $g=\sum_{k \in \mathbb{Z}^{n}} b_{k} e^{2 \pi i(k, x)}$ be trigonometric series. By the convolution of these series we mean the series

$$
f * g=\sum_{k \in \mathbb{Z}^{n}} a_{k} b_{k} e^{2 \pi i(k, x)}
$$

Note that for the «good» functions $f$ and $g$, the convolution defined by equality (2.1) coincides with the classical definition (1.1). If the functions $f$ and $g$ from the corresponding spaces in (1.3), then $f(x)=\sum_{k \in \mathbb{Z}^{n}} \hat{f}(k) e^{2 \pi i(k, x)}$ and $g(x) \stackrel{L_{\mathbf{r}}}{=} \sum_{k \in \mathbb{Z}^{n}} \hat{g}(k) e^{2 \pi i(k, x)}$ and $(f * g)(x)=\int_{\mathbb{T}^{n}} f(x-y) g(y) d y={ }^{L_{\mathbf{q}}} \sum_{k \in \mathbb{Z}^{n}} \hat{f}(k) \hat{g}(k) e^{2 \pi i(k, x)}$. Here, equalities are understood in the sense of the corresponding metrics.

We will need interpolation properties of anisotropic Sobolev and Besov spaces [24, 27]. Let $0<\boldsymbol{\theta}=$ $=\left(\theta_{1}, \ldots, \theta_{n}\right)<1, E=\left\{\varepsilon=\left(\varepsilon_{1} \ldots, \varepsilon_{n}\right): \varepsilon_{j} \in\{0,1\}, j=1, \ldots, n\right\}$ be the vertices of the $n$-dimensional unit cube, $\left\{A_{\varepsilon}\right\}_{\varepsilon \in E}$ be Banach spaces that are subspaces of some linear Hausdorff space. For the element $a \in \sum_{\varepsilon \in E} A_{\varepsilon}$, we define the functional

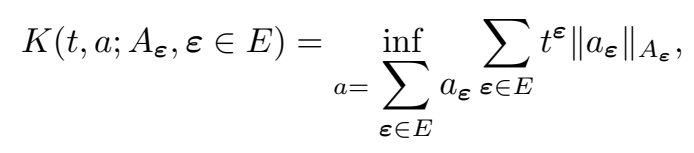

where $t^{\varepsilon}=t_{1}^{\varepsilon_{1}} \ldots t_{n}^{\varepsilon_{n}}$.

By $A_{\boldsymbol{\theta} \mathbf{q}}=\left(A_{\boldsymbol{\varepsilon}} ; \boldsymbol{\varepsilon} \in E\right)_{\boldsymbol{\theta} \mathbf{q}}$ we denote a linear subset of $\sum_{\boldsymbol{\varepsilon} \in E} A_{\boldsymbol{\varepsilon}}$, for elements of which

$$
\|a\|_{A_{\boldsymbol{\theta q}}}=\left(\int_{0}^{\infty} \ldots\left(\int_{0}^{\infty}\left|t_{1}^{\frac{1}{\theta_{1}}-1} \ldots t_{n}^{\frac{1}{\theta_{n}}-1} K\left(t, a ; A_{\varepsilon}, \varepsilon \in E\right)\right|^{q_{1}} \frac{d t_{1}}{t_{1}}\right)^{\frac{q_{2}}{q_{1}}} \ldots \frac{d t_{n}}{t_{n}}\right)^{\frac{1}{q_{n}}}<\infty
$$

is true. 
Lemma 2.1 [28] Let $T$ be a linear operator such that

$$
T: A_{\varepsilon} \rightarrow B_{\varepsilon} \text { with norm } M_{\varepsilon}, \varepsilon \in E .
$$

Then

with the norm $\|T\| \leq \max _{\varepsilon \in E} M_{\varepsilon}$.

$$
T:\left(A_{\boldsymbol{\varepsilon}} ; \boldsymbol{\varepsilon} \in E\right)_{\boldsymbol{\theta} \mathbf{q}} \rightarrow\left(B_{\boldsymbol{\varepsilon}}, \boldsymbol{\varepsilon} \in E\right)_{\boldsymbol{\theta} \mathbf{q}}
$$

Theorem 2.1 ([24]) Let $\mathbf{1} \leq \mathbf{p}=\left(p_{1}, \ldots, p_{n}\right)<\infty, \mathbf{0}<\mathbf{r}=\left(r_{1}, \ldots, r_{n}\right), \mathbf{q}=\left(q_{1}, \ldots, q_{n}\right) \leq \infty, \boldsymbol{\varepsilon} \in E$, $\boldsymbol{\alpha}_{0}=\left(\alpha_{1}^{0}, \ldots, \alpha_{n}^{0}\right), \boldsymbol{\alpha}_{1}=\left(\alpha_{1}^{1}, \ldots, \alpha_{n}^{1}\right) \in \mathbb{R}^{n}$. Then

$$
\begin{aligned}
& \left(B_{\mathbf{p r}}^{\boldsymbol{\alpha}_{\boldsymbol{\varepsilon}}}\left(\mathbb{T}^{n}\right) ; \varepsilon \in E\right)_{\boldsymbol{\theta} \mathbf{q}}=B_{\mathbf{p q}}^{\boldsymbol{\alpha}}\left(\mathbb{T}^{n}\right), \\
& \left(W_{\mathbf{p}}^{\boldsymbol{\alpha}_{\varepsilon}}\left(\mathbb{T}^{n}\right) ; \varepsilon \in E\right)_{\boldsymbol{\theta} \mathbf{q}}=B_{\mathbf{p q}}^{\boldsymbol{\alpha}}\left(\mathbb{T}^{n}\right),
\end{aligned}
$$

where $\boldsymbol{\alpha}_{\boldsymbol{\varepsilon}}=\left(\alpha_{1}^{\varepsilon}, \ldots, \alpha_{n}^{\varepsilon}\right), \mathbf{0}<\boldsymbol{\theta}=\left(\theta_{1}, \ldots, \theta_{n}\right)<\mathbf{1}, \boldsymbol{\alpha}=(1-\boldsymbol{\theta}) \boldsymbol{\alpha}_{0}+\boldsymbol{\theta} \boldsymbol{\alpha}_{1}$.

\section{Main result}

Lemma 3.1 Let $\mathbf{1} \leq \mathbf{q}, \mathbf{p}, \mathbf{r}<\infty, \frac{\mathbf{1}}{\mathbf{q}}+\mathbf{1}=\frac{\mathbf{1}}{\mathbf{p}}+\frac{\mathbf{1}}{\mathbf{r}}, \boldsymbol{\alpha}, \boldsymbol{\beta}, \boldsymbol{\gamma} \in \mathbb{R}^{n}, \boldsymbol{\alpha}=\boldsymbol{\beta}+\boldsymbol{\gamma}$. Suppose that $f \in W_{\mathbf{p}}^{\boldsymbol{\beta}}\left(\mathbb{T}^{n}\right)$, $g \in W_{\mathbf{r}}^{\boldsymbol{\gamma}}\left(\mathbb{T}^{n}\right)$. Then $f * g \in W_{\mathbf{q}}^{\boldsymbol{\alpha}}\left(\mathbb{T}^{n}\right)$ and

$$
\|f * g\|_{W_{\mathbf{q}}^{\boldsymbol{\alpha}}\left(\mathbb{T}^{n}\right)} \leq\|f\|_{W_{\mathbf{p}}^{\boldsymbol{\beta}}\left(\mathbb{T}^{n}\right)}\|g\|_{W_{\mathbf{r}}^{\boldsymbol{\gamma}}\left(\mathbb{T}^{n}\right)} .
$$

Proof. Let $f=\sum_{k \in \mathbb{Z}^{n}} a_{k} e^{2 \pi i(k, x)} \in W_{\mathbf{p}}^{\boldsymbol{\beta}}\left(\mathbb{T}^{n}\right), g=\sum_{k \in \mathbb{Z}^{n}} b_{k} e^{2 \pi i(k, x)} \in W_{\mathbf{r}}^{\boldsymbol{\gamma}}\left(\mathbb{T}^{n}\right)$. According to the definition, there are functions $f^{\boldsymbol{\beta}} \in L_{\mathbf{p}}\left(\mathbb{T}^{n}\right), g^{\boldsymbol{\gamma}} \in L_{\mathbf{r}}\left(\mathbb{T}^{n}\right)$ Fourier series of which coincide, respectively, with the $\sum_{k \in \mathbb{Z}^{n}} \bar{k}^{\boldsymbol{\beta}} a_{k} e^{2 \pi i(k, x)}, \sum_{k \in \mathbb{Z}^{n}} \bar{k}^{\boldsymbol{\gamma}} b_{k} e^{2 \pi i(k, x)}$.

From Young's inequality for Lebesgue spaces with mixed metric $[29 ; 25]\left(f^{\boldsymbol{\beta}} * g^{\boldsymbol{\gamma}}\right) \in L_{\mathbf{q}}\left(\mathbb{T}^{n}\right)$ and has the inequality

Now we note that

$$
\left\|f^{\boldsymbol{\beta}} * g^{\boldsymbol{\gamma}}\right\|_{L_{\mathbf{q}}\left(\mathbb{T}^{n}\right)} \leq\left\|f^{\boldsymbol{\beta}}\right\|_{L_{\mathbf{p}}\left(\mathbb{T}^{n}\right)}\left\|g^{\boldsymbol{\gamma}}\right\|_{L_{\mathbf{r}}\left(\mathbb{T}^{n}\right)}
$$

$$
\left(f^{\boldsymbol{\beta}} * g^{\boldsymbol{\gamma}}\right)(x) \stackrel{L_{\mathbf{q}}}{=} \sum_{k \in Z^{n}} \bar{k}^{\boldsymbol{\beta}+\boldsymbol{\gamma}} a_{k} b_{k} e^{2 \pi i(k, x)}=(f * g)^{\boldsymbol{\alpha}}(x)
$$

which means that $(f * g) \in W_{\mathbf{q}}^{\boldsymbol{\alpha}}\left(\mathbb{T}^{n}\right)$ and the inequality

$$
\|f * g\|_{W_{\mathbf{q}}^{\boldsymbol{\alpha}}\left(\mathbb{T}^{n}\right)} \leq\|f\|_{W_{\mathbf{p}}^{\boldsymbol{\beta}}\left(\mathbb{T}^{n}\right)}\|g\|_{W_{\mathbf{r}}^{\gamma}\left(\mathbb{T}^{n}\right)}
$$

holds.

Theorem 3.1 Let $\boldsymbol{\alpha}, \boldsymbol{\beta}, \boldsymbol{\gamma} \in \mathbb{R}^{n}, \boldsymbol{\alpha}=\boldsymbol{\beta}+\boldsymbol{\gamma}, \mathbf{1} \leq \mathbf{q}, \mathbf{p}, \mathbf{r}<\infty, \mathbf{1}+\frac{\mathbf{1}}{\mathbf{q}}=\frac{\mathbf{1}}{\mathbf{p}}+\frac{\mathbf{1}}{\mathbf{r}}, \mathbf{0}<\mathbf{h}, \boldsymbol{\eta}, \boldsymbol{\xi} \leq \infty, \frac{\mathbf{1}}{\mathbf{h}}=\frac{\mathbf{1}}{\boldsymbol{\eta}}+\frac{\mathbf{1}}{\boldsymbol{\xi}}$. Suppose that $f \in B_{\mathbf{p} \boldsymbol{\eta}}^{\boldsymbol{\beta}}\left(\mathbb{T}^{n}\right), g \in B_{\mathbf{r} \boldsymbol{\xi}}^{\boldsymbol{\gamma}}\left(\mathbb{T}^{n}\right)$. Then $f * g \in B_{\mathbf{q h}}^{\boldsymbol{\alpha}}\left(\mathbb{T}^{n}\right)$ and

$$
\|f * g\|_{B_{\mathbf{q} h}^{\alpha}\left(\mathbb{T}^{n}\right)} \leq C\|f\|_{B_{\mathbf{p} \eta}^{\beta}\left(\mathbb{T}^{n}\right)}\|g\|_{B_{\mathbf{r} \xi}^{\gamma}\left(\mathbb{T}^{n}\right)} .
$$

Proof. Let $f \in W_{\mathbf{p}}^{\boldsymbol{\beta}}\left(\mathbb{T}^{n}\right), g \in W_{\mathbf{r}}^{\boldsymbol{\gamma}}\left(\mathbb{T}^{n}\right)$, then from Lemma 3.1 it follows that $(f * g) \in W_{\mathbf{q}}^{\boldsymbol{\alpha}}\left(\mathbb{T}^{n}\right)$ and the following inequality

$$
\|f * g\|_{W_{\mathbf{q}}^{\boldsymbol{\alpha}}\left(\mathbb{T}^{n}\right)} \leq\|f\|_{W_{\mathbf{p}}^{\boldsymbol{\beta}}\left(\mathbb{T}^{n}\right)}\|g\|_{W_{\mathbf{r}}^{\gamma}\left(\mathbb{T}^{n}\right)}
$$

holds true.

Let $\boldsymbol{\alpha}_{0}=\left(\alpha_{1}^{0}, \ldots, \alpha_{n}^{0}\right), \boldsymbol{\alpha}_{1}=\left(\alpha_{1}^{1}, \ldots, \alpha_{n}^{1}\right), \boldsymbol{\beta}_{0}=\left(\beta_{1}^{0}, \ldots, \beta_{n}^{0}\right), \boldsymbol{\beta}_{1}=\left(\beta_{1}^{1}, \ldots, \beta_{n}^{1}\right) \in \mathbb{R}^{n}, \alpha_{i}^{0} \neq \alpha_{i}^{1}, \beta_{i}^{0} \neq \beta_{i}^{1}$, $i=\overline{1, n}$. Let $\boldsymbol{\alpha}_{\varepsilon}=\left(\alpha_{1}^{\varepsilon_{1}}, \ldots, \alpha_{n}^{\varepsilon_{n}}\right), \boldsymbol{\beta}_{\varepsilon}=\left(\beta_{1}^{\varepsilon_{1}}, \ldots, \beta_{n}^{\varepsilon_{n}}\right), \boldsymbol{\varepsilon} \in E$ such that $\boldsymbol{\alpha}_{\varepsilon}-\boldsymbol{\beta}_{\boldsymbol{\varepsilon}}=\boldsymbol{\gamma}$.

We rewrite inequality (3.1) for the $\boldsymbol{\alpha}_{\varepsilon}$ and $\boldsymbol{\beta}_{\boldsymbol{\varepsilon}}$ parameters

$$
\|f * g\|_{W_{\mathbf{q}}^{\boldsymbol{\alpha}_{\boldsymbol{\varepsilon}}\left(\mathbb{T}^{n}\right)}} \leq\|f\|_{W_{\mathbf{p}}^{\boldsymbol{\beta}_{\boldsymbol{\varepsilon}}\left(\mathbb{T}^{n}\right)}}\|g\|_{W_{\mathbf{r}}^{\gamma}\left(\mathbb{T}^{n}\right)}, \boldsymbol{\alpha}_{\varepsilon}=\boldsymbol{\beta}_{\boldsymbol{\varepsilon}}+\gamma, \boldsymbol{\varepsilon} \in E .
$$


For a fixed $g \in W_{\mathbf{r}}^{\gamma}\left(\mathbb{T}^{n}\right)$ operator $A_{g} f=f * g$ acts boundedly from $W_{\mathbf{p}}^{\boldsymbol{\beta}_{\varepsilon}}\left(\mathbb{T}^{n}\right)$ to $W_{\mathbf{q}}^{\boldsymbol{\alpha}_{\varepsilon}}\left(\mathbb{T}^{n}\right)$. Then, using the anisotoropic interpolation theorem (Theorem 2.1)

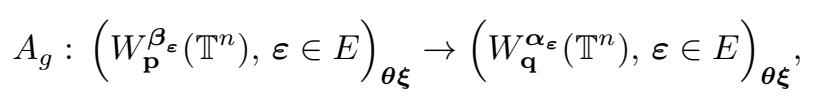

we obtain that the operator acts boundedly

$$
A_{g}: B_{\mathbf{p} \xi}^{\beta}\left(\mathbb{T}^{n}\right) \rightarrow B_{\mathbf{q} \xi}^{\alpha}\left(\mathbb{T}^{n}\right)
$$

and

$$
\left\|A_{g}\right\| \leq C\|g\|_{W_{\mathbf{r}}^{\gamma}\left(\mathbb{T}^{n}\right)},
$$

where $\boldsymbol{\alpha}=(1-\boldsymbol{\theta}) \boldsymbol{\alpha}_{0}+\boldsymbol{\theta} \boldsymbol{\alpha}_{1}, \boldsymbol{\beta}=(1-\boldsymbol{\theta}) \boldsymbol{\beta}_{0}+\boldsymbol{\theta} \boldsymbol{\beta}_{1}$ for any $\mathbf{0}<\boldsymbol{\theta}=\left(\theta_{1}, \ldots, \theta_{n}\right)<\mathbf{1}$. Thus, we have obtained the inequality:

$$
\|f * g\|_{B_{\mathbf{q} \xi}^{\alpha}\left(\mathbb{T}^{n}\right)} \leq C\|f\|_{B_{\mathbf{p} \xi}^{\beta}\left(\mathbb{T}^{n}\right)}\|g\|_{W_{\mathbf{r}}^{\gamma}\left(\mathbb{T}^{n}\right)},
$$

where $\boldsymbol{\alpha}, \boldsymbol{\beta}, \boldsymbol{\gamma}, \mathbf{p}, \mathbf{q}, \mathbf{r}$ satisfy the conditions of the theorem.

In inequality (3.2) we assume that $\boldsymbol{\xi}=\infty$, then we have

$$
\|f * g\|_{B_{\mathbf{q} \infty}^{\alpha}\left(\mathbb{T}^{n}\right)} \leq C\|f\|_{B_{\mathbf{p} \infty}^{\beta}\left(\mathbb{T}^{n}\right)}\|g\|_{W_{\mathbf{r}}^{\gamma}\left(\mathbb{T}^{n}\right)} .
$$

We fix $\boldsymbol{\beta} \in \mathbb{R}^{n}$. Let $\boldsymbol{\alpha}_{0}, \boldsymbol{\alpha}_{1}, \gamma_{0}, \gamma_{1} \in \mathbb{R}^{n}$ be arbitrary vector parameters satisfying the conditions $\boldsymbol{\alpha}_{i}=\boldsymbol{\beta}+\boldsymbol{\gamma}_{i}$, $i=0,1$ и $\alpha_{j}^{0} \neq \alpha_{j}^{1}, \gamma_{j}^{0} \neq \gamma_{j}^{1}, j=\overline{1, n}$. Then for the parameters $\boldsymbol{\alpha}_{\varepsilon}=\left(\alpha_{1}^{\varepsilon_{1}}, \ldots \alpha_{n}^{\varepsilon_{n}}\right), \gamma_{\varepsilon}=\left(\gamma_{1}^{\varepsilon_{1}}, \ldots \gamma_{n}^{\varepsilon_{n}}\right), \varepsilon \in E$ the inequality

$$
\|f * g\|_{B_{\mathbf{q} \infty}^{\alpha_{\varepsilon}\left(\mathbb{T}^{n}\right)}} \leq C\|f\|_{B_{\mathbf{p} \infty}^{\beta}\left(\mathbb{T}^{n}\right)}\|g\|_{W_{\mathbf{r}}^{\gamma_{\boldsymbol{\varepsilon}}\left(\mathbb{T}^{n}\right)}} .
$$

holds.

Now for a fixed $f(x)$ we define a linear operator $B_{f} g=f * g$. Then $B_{f}$ acts boundedly from $W_{\mathbf{r}}^{\gamma_{\varepsilon}}\left(\mathbb{T}^{n}\right)$ in $B_{\mathbf{q} \infty}^{\alpha_{\varepsilon}}\left(\mathbb{T}^{n}\right)$ with an estimate of the norm

$$
\left\|B_{f}\right\|_{W_{\mathbf{r}}^{\gamma_{\varepsilon}}\left(\mathbb{T}^{n}\right) \rightarrow B_{\mathbf{q} \infty}^{\alpha_{\infty}}\left(\mathbb{T}^{n}\right)} \leq C\|f\|_{B_{\mathbf{p} \infty}^{\beta}\left(\mathbb{T}^{n}\right)} .
$$

Further, using Theorem 2.1 and Lemma 2.1, we have that the operator $B_{f}$ is bounded from $B_{\mathbf{r} \xi}^{\gamma}\left(\mathbb{T}^{n}\right)$ to $B_{\mathbf{q} \xi}^{\alpha}\left(\mathbb{T}^{n}\right)$ and

$$
\|f * g\|_{B_{\mathbf{q} \xi}^{\alpha}\left(\mathbb{T}^{n}\right)} \leq C\|f\|_{B_{\mathbf{p} \infty}^{\beta}\left(\mathbb{T}^{n}\right)}\|g\|_{B_{\mathbf{r} \xi}^{\gamma}\left(\mathbb{T}^{n}\right)},
$$

where $\boldsymbol{\alpha}=(1-\boldsymbol{\theta}) \boldsymbol{\alpha}_{0}+\boldsymbol{\theta} \boldsymbol{\alpha}_{1}, \boldsymbol{\gamma}=(1-\boldsymbol{\theta}) \boldsymbol{\gamma}_{0}+\boldsymbol{\theta} \boldsymbol{\gamma}_{1}$ for any $\mathbf{0}<\boldsymbol{\theta}=\left(\theta_{0}, \ldots, \theta_{n}\right)<\mathbf{1}$.

Similarly, we can obtain the inequality

$$
\|f * g\|_{B_{\mathbf{q} \eta}^{\alpha}\left(\mathbb{T}^{n}\right)} \leq C\|f\|_{B_{\mathbf{p} \eta}^{\beta}\left(\mathbb{T}^{n}\right)}\|g\|_{B_{\mathbf{r}^{\infty}\left(\mathbb{T}^{n}\right)}^{\gamma}} .
$$

Thus, for the bilinear convolution operator $T(f, g)=f * g$, we have

$$
\begin{aligned}
& T: B_{\mathbf{p} \infty}^{\boldsymbol{\beta}_{0}}\left(\mathbb{T}^{n}\right) \times B_{\mathbf{r} \xi}^{\gamma_{0}}\left(\mathbb{T}^{n}\right) \rightarrow B_{\mathbf{q} \xi}^{\boldsymbol{\alpha}_{0}}\left(\mathbb{T}^{n}\right), \\
& T: B_{\mathbf{p} \boldsymbol{\eta}}^{\boldsymbol{\beta}_{1}}\left(\mathbb{T}^{n}\right) \times B_{\mathbf{r} \infty}^{\gamma_{1}}\left(\mathbb{T}^{n}\right) \rightarrow B_{\mathbf{q} \boldsymbol{\eta}}^{\boldsymbol{\alpha}_{1}}\left(\mathbb{T}^{n}\right),
\end{aligned}
$$

where the corresponding parameters satisfy the conditions of the theorem.

Next, applying the bilinear interpolation theorem (Theorem 4.4.1, [30, 125]), we have

$$
T:\left(B_{\mathbf{p} \infty}^{\boldsymbol{\beta}_{0}}\left(\mathbb{T}^{n}\right), B_{\mathbf{p} \boldsymbol{\eta}}^{\boldsymbol{\beta}_{1}}\left(\mathbb{T}^{n}\right)\right)_{[\theta]} \times\left(B_{\mathbf{r} \xi}^{\gamma_{0}}\left(\mathbb{T}^{n}\right), B_{\mathbf{r} \infty}^{\gamma_{1}}\left(\mathbb{T}^{n}\right)\right)_{[\theta]} \rightarrow\left(B_{\mathbf{q} \xi}^{\boldsymbol{\alpha}_{0}}\left(\mathbb{T}^{n}\right), B_{\mathbf{q} \boldsymbol{\eta}}^{\boldsymbol{\alpha}_{1}}\left(\mathbb{T}^{n}\right)\right)_{[\theta]} .
$$

Since the space $B_{\mathbf{p q}}^{\mathbf{s}}\left(\mathbb{T}^{n}\right)$ is a retract of $l_{\mathbf{q}}^{\mathbf{s}}\left(L_{\mathbf{p}}\right)\left(\mathbb{T}^{n}\right)$, we have

$$
\left(B_{\mathbf{p s}_{0}}^{\boldsymbol{\beta}_{0}}\left(\mathbb{T}^{n}\right), B_{\mathbf{p s}_{1}}^{\boldsymbol{\beta}_{1}}\left(\mathbb{T}^{n}\right)\right)_{[\theta]}=B_{\mathbf{p s}}^{\boldsymbol{\beta}}\left(\mathbb{T}^{n}\right),
$$

where $\boldsymbol{\beta}=\boldsymbol{\beta}_{0}(1-\boldsymbol{\theta})+\boldsymbol{\beta}_{1} \boldsymbol{\theta}, \frac{1}{\mathrm{~s}}=\frac{1-\boldsymbol{\theta}}{\mathrm{s}_{\mathbf{0}}}+\frac{\boldsymbol{\theta}}{\mathrm{s}_{1}}$. 
This implies

$$
T: B_{\mathbf{p} \boldsymbol{\eta}}^{\boldsymbol{\beta}}\left(\mathbb{T}^{n}\right) \times B_{\mathbf{r} \xi}^{\gamma}\left(\mathbb{T}^{n}\right) \rightarrow B_{\mathbf{q h}}^{\boldsymbol{\alpha}}\left(\mathbb{T}^{n}\right) .
$$

Finally,

where

$$
\|f * g\|_{B_{\mathbf{q} \mathbf{h}}^{\alpha}\left(\mathbb{T}^{n}\right)} \leq C\|f\|_{B_{\mathbf{p} \eta}^{\beta}\left(\mathbb{T}^{n}\right)}\|g\|_{B_{\mathbf{r} \xi}^{\gamma}\left(\mathbb{T}^{n}\right)},
$$

$$
\frac{1}{\mathrm{~h}}=\frac{1}{\eta}+\frac{1}{\xi}, 1+\frac{1}{\mathrm{q}}=\frac{1}{\mathrm{p}}+\frac{1}{\mathrm{r}}, \alpha=\beta+\gamma .
$$

Taking into account the embeddings of spaces ([24], Theorem 4), we can obtain the following theorem.

Theorem 3.2 Let $\boldsymbol{\alpha}, \boldsymbol{\beta}, \boldsymbol{\gamma} \in \mathbb{R}^{n}, \boldsymbol{\alpha} \leq \boldsymbol{\beta}+\boldsymbol{\gamma}, 1 \leq \mathbf{q}, \mathbf{p}, \mathbf{r}<\infty, \boldsymbol{\alpha}=\boldsymbol{\beta}+\boldsymbol{\gamma}+\mathbf{1}+\frac{\mathbf{1}}{\mathbf{q}}-\frac{\mathbf{1}}{\mathbf{p}}-\frac{\mathbf{1}}{\mathbf{r}}, \mathbf{0}<\mathbf{h}, \boldsymbol{\eta}, \boldsymbol{\xi} \leq \infty$. Suppose that $f(x)$ and $g(x)$ are measurable functions on $\mathbb{T}^{n}$ such that $f \in B_{\mathbf{p} \boldsymbol{\eta}}^{\boldsymbol{\beta}}\left(\mathbb{T}^{n}\right), g \in B_{\mathbf{r} \boldsymbol{\xi}}^{\gamma}\left(\mathbb{T}^{n}\right)$. Then $f * g \in B_{\mathbf{q h}}^{\alpha}\left(\mathbb{T}^{n}\right)$ and

$$
\|f * g\|_{B_{\mathbf{q} h}^{\alpha}\left(\mathbb{T}^{n}\right)}^{\alpha} \leq C\|f\|_{B_{\mathbf{p} \eta}^{\beta}\left(\mathbb{T}^{n}\right)}\|g\|_{B_{\mathbf{r} \xi}^{\gamma}\left(\mathbb{T}^{n}\right)},
$$

where $\frac{1}{\mathbf{h}} \leq \frac{1}{\eta}+\frac{1}{\xi}$

\section{References}

1 Bennett C. Interpolation of Operators / C. Bennett, R. Sharpley. Pure and Applied Mathematics 129, Boston, MA, Academic Press, INC. - 1988. - 469 p.

2 Brézis H. A note on limiting cases of Sobolev embeddings and convolution inequalities / H. Brézis, S. Wainger // Comm. Partial Differential Equations. - 1980. - 5. - No. 7. - P. 773-789.

3 Hörmander L. The analysis of linear partial differential operators I. Distribution theory and Fourier analysis. Reprint of the second edition / L. Hörmander. - Berlin: Classics in Mathematics, SpringerVerlag. - 2013. - $440 \mathrm{p}$.

4 O'Neil R. Convolution operators and $L(p, q)$ spaces / R. O'Neil // Duke Math. J. - 1963. - 30. P. 129-142.

5 Yap, L.Y.H. Some remarks on convolution operators and $l(p, q)$ spaces / L.Y.H. Yap // Duke Math J. 1969. - 36. - P. 647-658.

6 Hunt R.A. On $L(p, q)$ spaces / R.A. Hunt // Enseignement Math. - 1966. - 12. - No. 2. - P. 249-276.

7 Nursultanov E. Convolution inequalities in Lorentz spaces / E. Nursultanov, S. Tikhonov // J. Fourier Anal. Appl. - 2011. - 17. - P. 486-505.

8 Blozinski A.P. On a convolution theorem for $L(p, q)$ spaces / A.P. Blozinski // Trans. Amer. Math. Soc. - 1972. - 164. - P. 255-265.

9 Нурсултанов Е.Д. Мультипликаторы кратных рядов Фурье / Е.Д. Нурсултанов, Н.Т. Тлеуханова // Тр. МИ РАН им. В.А. Стеклова. - 1999. - 227. - Р. 231-236.

10 Tleukhanova N.T. The O'Neil-type inequalities for anisotropic Lorentz spaces $L(\bar{p}, \bar{q}) /$ N.T. Tleukhanova, K.K. Sadykova // Eurasian Mathematical Journal. В печати.

11 Nursultanov E. Norm inequalities for convolution operators / E. Nursultanov, S. Tikhonov, N. Tleukhanova // C. R. Acad. Sci. Paris. - 2009. - I. - No. 347. - P. 1385-1388.

12 Nursultanov E. Norm convolution inequalities in Lebesgue spaces / E. Nursultanov, S. Tikhonov, N. Tleukhanova // Rev. Mat. Iberoam. - 2018. - 34. - No. 2. - P. 811-838.

13 Heil C. An introduction to weighted Wiener amalgams. In Wavelets and their applications / C. Heil // Allied Publishers, New Delphi. - 2003. - P. 183-216.

14 Kamińska A. On convolution operator in Orlicz spaces / A. Kamińska, J. Musielak // Rev. Mat. Univ. Complutense. - 1989. - 2. - P. 157-178.

15 Kerman R.A. Convolution theorems with weights / R.A. Kerman // Trans. Amer. Math. Soc. - 1983. 280. - No. 1. - P. 207-219.

16 Kerman R. Convolution algebras with weighted rearrangement-invariant norm / R. Kerman, E. Sawyer // Studia Math. - 1994. - 108. - No. 2. - P. 103-126. 
17 Nursultanov E. Weighted norm inequalities for convolution and Riesz potential / E. Nursultanov, S. Tikhonov // Potential Analysis. - 2015. - 42. - No. 2. - P. 435-456.

18 Sampson G. $\left(L_{p}, L_{q}\right)$ mapping properties of convolution transforms / G. Sampson, A. Naparstek, V. Drobot // Studia Math. - 1976. - 55. - No. 1. - P. 41-70.

19 Головкин К.К. Оценки интегральных операторов в трансляционно-инвариантных нормах / K.K. Головкин, В.А. Солонников // Тр. МИ АН СССР. - 1964. - 70. - С. 47-58.

20 Головкин К.К. Оценки интегральных операторов в трансляционно-инвариантных нормах. II / K.К. Головкин, В.А. Солонников // Тр. МИ АН СССР. - 1966. - 92. - С. 5-30.

21 Батыров Б.Е. Об оценках сверток в пространствах Никольского-Бесова / Б.Е. Батыров, В.И. Буренков // Док. АН СССР. - 1993. - 330. - № 1. - С. 9-11.

22 Bui H. Weighted Young's inequality and convolution theorems on weighted Besov spaces / H. Bui // Math. Nachr. - 1994. - 170. - P. 25-37.

23 Головкин К.К. Об оценках операторов свертки / К.К. Головкин, В.А. Солонников // Зап. науч. сем. ЛОМИ. - 1968. - 7. - Р. 6-86.

24 Nursultanov E.D. Interpolation theorems for anisotropic function spaces and their applications / E.D. Nursultanov // Dokl. Math. - 2004. - 69. - No. 1. - P. 16--19.

25 Бекмаганбетов К.А. Теоремы вложения анизотропных пространств Бесова $B_{\mathbf{p r}}^{\alpha \mathbf{q}}\left([0,2 \pi)^{n}\right) /$ K.A. Бекмаганбетов, Е.Д. Нурсултанов // Изв. РАН. Сер. Матем. - 2009. - 73. - № 4. - Р. 3-16.

26 Bekmaganbetov K.A. Order of the orthoprojection widths of the anisotropic Nikolsky-Besov classes in the anisotropic Lorentz space / K.A. Bekmaganbetov, Ye. Toleugazy // Eurasian Math. J. - 2016. - 7. - No. 3. - P. 8-16.

27 Nursultanov E.D. Application of Interpolational Methods to the Study of Properties of Functions of Several Variables / E.D. Nursultanov // Mathematical Notes. - 2004. - 75. - No. 3. - P. 341-351.

28 Nursultanov E.D. On the coefficients of multiple Fourier series in $L_{p}$ spaces / E.D. Nursultanov // Izvestiia RAN: Ser. Mat. - 2000. - 64. - No. 1. - P. 95-122.

29 Бесов О.В. Интегральные представления функций и теоремы вложения / О.В. Бесов, В.П. Ильин, С.М. Никольский. - М.: Наука, 1975. - 480 с.

30 Берг Й. Интерполяционные пространства. Введение / Й. Берг, Й. Лефстрем. - М.: Мир, 1980. 264 c.

\author{
К.К. Садыкова, Н.Т. Тлеуханова
}

\title{
Аралас туындысы басым анизотропты Бесов кеңістігіндегі үйірткі операторының нормаларын бағалау
}

\begin{abstract}
Мақалада үйірткі операторы нормасының аралас туындысы басым Соболев және анизотропты Никольский-Бесов кеңістіктеріндегі шенелуі зерттелді. Аралас туындысы басым Соболев кеңістігі үшін Юнг теңсіздігінің аналогы алынды, атап айтқанда, $W_{p}^{\gamma} * W_{r}^{\beta} \hookrightarrow W_{q}^{\alpha}$ түріндігі қатынас дәлелденді, мұнда катынас параметрлеріне сәйкесінше шарттар қойылған. Жұмыстың негізгі мақсаты келесі есеп болып табылады: айталық, $f$ және $g$ - Никольский-Бесов кеңістігі шкаласының қандай да бір функциялар класы болсын. Олардың $f * g$ үйірткісі қай кеңістікке жататынын анықтау қажет. (1) қатынас пен Нұрсұлтановтың анизотропты кеңістіктерге арналған интерполяциялық теоремаларын қолдана отырып, $B_{p q}^{\boldsymbol{\alpha}}$ Никольский-Бесов кеңістігінің шкаласы үшін О'Нейл теоремасының аналогы алынды, мұнда $\alpha, p, q-$ векторлық параметрлер. $B_{p s_{1}}^{\gamma} * B_{r s_{2}}^{\beta} \hookrightarrow B_{q s}^{\alpha}$ түріндігі қатынас дәлелденді, мұнда қатынас параметрлеріне сәйкесінше шарттар қойылған. Осы жұмыста алынған теоремалар изотропты Никольский-Бесов кеңістігіндегі, яғни скаляр параметрлі кеңістіктегі, Батыров пен Буренковтың ұқсас есептер қарастырган нәтижелерін толықтырады.
\end{abstract}

Kiлm сөздер: үйірткі операторы, анизотропты Бесов кеңістігі, анизотропты Соболев кеңістігі, интерполяция. 
К.К. Садыкова, Н.Т. Тлеуханова

\title{
Оценки нормы оператора свертки в анизотропных пространствах Бесова с доминирующей смешанной производной
}

\begin{abstract}
В статье исследована ограниченность нормы оператора свертки в пространствах Соболева, с доминирующей смешанной производной, и анизотропных пространствах Никольского-Бесова. Для пространств Соболева с доминирующей смешанной производной получен аналог неравенства Юнга, а именно доказаны соотношения вида $W_{p}^{\gamma} * W_{r}^{\beta} \hookrightarrow W_{q}^{\alpha}$ при выполнении соответствующих условий на параметры. Основной целью работы является решение следующей задачи: пусть $f$ и $g-$ функции из некоторых классов шкалы пространств Никольского-Бесова. Нужно определить, к какому пространству принадлежит их свертка $f * g$. Используя соотношение $(1)$ и интерполяционные теоремы Нурсултанова для анизотропных пространств, получен аналог теоремы О'Нейла для шкалы пространств Никольского-Бесова $B_{p q}^{\alpha}$, где $\alpha, p, q$ - векторные параметры. Получены соотношения вида $B_{p s_{1}}^{\gamma} * B_{r s_{2}}^{\beta} \hookrightarrow B_{q s}^{\alpha}$, при соответствующих соотношениях векторных параметров. Полученные в данной работе теоремы дополняют результаты Батырова и Буренкова, где рассматривались подобные задачи в изотропных пространствах Никольского-Бесова, т.е. в пространствах, где параметры являются скалярами.
\end{abstract}

Ключевые слова: оператор свертки, анизотропные пространства Бесова, анизотропные пространства Соболева, интерполяция.

\section{References}

1 Bennett, C. \& Sharpley, R. (1988). Interpolation of Operators. Pure and Applied Mathematics 129, Boston, MA, Academic Press, INC.

2 Brézis, H. \& Wainger, S. (1980). A note on limiting cases of Sobolev embeddings and convolution inequalities. Comm. Partial Differential Equations, 5, 7, 773-789.

3 Hörmander, L. (2013). The analysis of linear partial differential operators I. Distribution theory and Fourier analysis. Reprint of the second edition. Berlin: Classics in Mathematics, Springer-Verlag.

4 O'Neil, R. (1963). Convolution operators and L(p,q) spaces. Duke Math. J., 30, 129-142.

5 Yap, L.Y.H. (1969). Some remarks on convolution operators and $l(p, q)$ spaces. Duke Math J., 36, $647-658$.

6 Hunt, R.A. (1966). On $L(p, q)$ spaces. Enseignement Math., 12, 2, 249-276.

7 Nursultanov, E. \& Tikhonov, S. (2011). Convolution inequalities in Lorentz spaces. J. Fourier Anal. Appl., 17, 486-505.

8 Blozinski, A.P. (1972). On a convolution theorem for $L(p, q)$ spaces. Trans. Amer. Math. Soc., 164, 255265.

9 Nursultanov, E.D. \& Tleukhanova, N.T. (1999). Multiplikatory kratnykh riadov Fure [Multipliers of multiple Fourier series]. Trudy MI RAN imeni V.A. Steklova - Proceedings of the Steklov MI RAS, 227, 231-236 [in Russian].

10 Tleukhanova, N.T. \& Sadykova, K.K. The O'Neil-type inequalities for anisotropic Lorentz $\operatorname{spaces} L(\bar{p}, \bar{q})$. Eurasian Mathematical Journal.

11 Nursultanov, E. \& Tikhonov, S. \& Tleukhanova, N. (2009). Norm inequalities for convolution operators. C. R. Acad. Sci. Paris, I, 347, 1385-1388.

12 Nursultanov, E. \& Tikhonov, S. \& Tleukhanova, N. (2018). Norm convolution inequalities in Lebesgue spaces. Rev. Mat. Iberoam., 34, 2, 811-838.

13 Heil, C. (2003). An introduction to weighted Wiener amalgams. In Wavelets and their applications. Allied Publishers, New Delphi, 183-216.

14 Kamińska, A. \& Musielak, J. (1989). On convolution operator in Orlicz spaces. Rev. Mat. Univ. Complutense, 2, 157-178.

15 Kerman, R.A. (1983). Convolution theorems with weights. Trans. Amer. Math. Soc., 280, 1, $207-219$. 
16 Kerman, R. \& Sawyer, E. (1994). Convolution algebras with weighted rearrangement-invariant norm. Studia Math., 108, 2, 103-126.

17 Nursultanov, E. \& Tikhonov, S. (2015). Weighted norm inequalities for convolution and Riesz potential. Potential Analysis, 42, 2, 435-456.

18 Sampson, G. \& Naparstek, A. \& Drobot, V. (1976). $\left(L_{p}, L_{q}\right)$ mapping properties of convolution transforms. Studia Math., 55, 1, 41-70.

19 Golovkin, K.K. \& Solonnikov, V.A. (1964). Otsenki intehralnykh operatorov v transliatsionno-invariantnykh normakh [Estimates of integral operators in translation-invariant norms]. Trudy MI AN SSSRProceedings of MI AN USSR, 70, 47-58 [in Russian].

20 Golovkin, K.K. \& Solonnikov, V.A. (1966). Otsenki intehralnykh operatorov v transliatsionno-invariantnykh normakh. II [Estimates of integral operators in translation-invariant norms. II]. Trudy MI AN SSSR - Proceedings of MI AN USSR, 92, 5-30 [in Russian].

21 Batyrov, B.E. \& Burenkov, V.I. (1993). Ob otsenkakh svertok v prostranstvakh Nikolskoho-Besova [On estimates of convolutions in Nikolsky-Besov spaces]. Doklady AN SSSR - Reports of the AS USSR, 330, 1, 9-11 [in Russian].

22 Bui, H. (1994). Weighted Young's inequality and convolution theorems on weighted Besov spaces. Math. Nachr., 170, 25-37.

23 Golovkin, K.K., \& Solonnikov, V.A. (1968). Ob otsenkakh operatorov svertki [On estimates of convolution operators]. Zapiski nauchnoho seminara LOMI - Notes of scientific seminars of the LBMI, 7, 6-86 [in Russian].

24 Nursultanov, E.D. (2004). Interpolation theorems for anisotropic function spaces and their applications. Dokl. Math., 69, 1, 16-19.

25 Bekmaganbetov, K.A. \& Nursultanov, E.D. (2009). Teoremy vlozheniia anizotropnykh prostranstv Besova $B_{\mathbf{p r}}^{\alpha \mathbf{q}}\left([0,2 \pi)^{n}\right)$ [Embedding theorems for anisotropic Besov spaces $\left.B_{\mathbf{p r}}^{\alpha \mathbf{q}}\left([0,2 \pi)^{n}\right)\right]$. Izvestiia RAN SSSR. Seriia Matematila - Proceedings of the RAS USSR. Ser. Math., 73, 4, 3-16 [in Russian].

26 Bekmaganbetov, K.A. \& Toleugazy, Ye. (2016). Order of the orthoprojection widths of the anisotropic Nikolsky-Besov classes in the anisotropic Lorentz space. Eurasian Math. J., 7, 3, 8-16.

27 Nursultanov, E.D. (2004). Application of Interpolational Methods to the Study of Properties of Functions of Several Variables. Mathematical Notes, 75, 3, 341-351.

28 Nursultanov, E.D. (2000). On the coefficients of multiple Fourier series in $L_{p}$ spaces. Izvestiia RAN: Ser. Mat., 64, 1, 95-122.

29 Besov, O.V. \& Il'in, V.P. \& Nikolskii, S.M. (1975). Intehralnye predstavleniia funktsii i teoremy vlozheniia [Integral presentation of functions and theorem of embanding]. Moscow: Nauka [in Russian].

30 Berg, I. \& Löfstrem, I. (1980) Interpoliatsionnye prostranstva. Vvedenie [Interpolation spaces. Introduction]. Moscow: Mir [in Russian]. 